\title{
Al Qaida et l'État islamique : histoires croisées
}

\section{Mohammad-Mahmoud Ould Mohamedou}

\section{OpenEdition}

\section{Journals}

Édition électronique

URL : https://journals.openedition.org/annuaire-cdf/16467

DOI : $10.4000 /$ annuaire-cdf. 16467

ISBN : 978-2-7226-0572-5

ISSN : 2109-9227

Éditeur

Collège de France

Édition imprimée

Date de publication : 30 décembre 2020

Pagination : 713-715

ISBN : 978-2-7226-0516-9

ISSN : 0069-5580

Référence électronique

Mohammad-Mahmoud Ould Mohamedou, «Al Qaida et l'État islamique : histoires croisées »,

L'annuaire du Collège de France [En ligne], 118 | 2020, mis en ligne le 01 avril 2021, consulté le 22 août

2022. URL : http://journals.openedition.org/annuaire-cdf/16467 ; DOI : https://doi.org/10.4000/

annuaire-cdf. 16467 


\title{
AL QAIDA ET L'ÉTAT ISLAMIQUE : HISTOIRES CROISÉES
}

\author{
Mohammad-Mahmoud OULD MOHAMEDOU \\ Professeur d'histoire internationale au Graduate Institute à Genève (Suisse)
}

Mots-clés : Al Qaida, État islamique

Conférence prononcée le 29 novembre 2017 (invitation sur proposition du Pr Henry Laurens), disponible en audio et vidéo sur le site internet du Collège de France : https://www.college-de-france.fr/site/henry-laurens/guestlecturer-2017-2018.htm.

Le projet d'histoire internationale et politique présenté ici interroge de façon critique l'historiographie des mouvements non étatiques transnationaux $\mathrm{Al}$ Qaida et l'organisation de l'État islamique (EI), invitant une réflexion plus large sur la nature de la violence qu'ils projettent. Cette réflexion fait explicitement rupture avec les lectures dominantes dans les sciences sociales occidentales qui situent ces mouvements dans des dimensions principalement régionales et religieuses. Désindexant ces deux biais, les questions alternatives posées sont de nature métahistorique et politique. Loin des approches exceptionalisant l'actualité de ces groupes et des régions du monde dont ils proviennent s'impose plus pertinemment la triple notion de circulation, d'interconnexion et d'interdépendance de leur violence. L'auto-capacitation de cette nouvelle génération de groupes armés desserre la dimension spatiale et consacre la transnationalisation grandissante des relations internationales.

Dans ce contexte, l'avènement de l'EI s'inscrit dans un parcours de violence politique mondialisée plus vaste entamé plus tôt par une autre organisation, Al Qaida. Les micro-récits relatifs à ces deux groupes centrés sur leurs leaders et leur religiosité théâtralisée ont néanmoins occulté les enseignements plus larges qu'il faut tirer concernant l'histoire contemporaine de la violence politique elle-même. L'EI, comme Al Qaida, souffre un déficit analytique et une absence de conceptualisation. Les sciences sociales, généralement, et les relations internationales, plus précisément, ont, pour l'heure, été incapables de cerner la nature des importantes transformations systémiques - la militarisation de l'islamisme, l'indigénisation du transnationalisme et l'entreprenariat de violence post-moderne - introduites par ces deux groupes. Confinées à des approches sécuritaires, informées de façon culturaliste et distillées sur un mode médiatique, les analyses de ces nouvelles formes de violences dénotent un manque conceptuel, alors que le terrorisme est aujourd'hui avant tout révélateur d'un «moment historique » de ces mutations de la contestation, dont l'essor a lieu sur l'arrière-plan d'une modernisation rapide et mondialisée. Le résultat de cette évolution, qui fait le lit de l'État islamique, se décline à partir de la fin des années 2000, et le terrorisme post-Al Qaida se révèle être principalement l'introduction de la privatisation du terrorisme dont les cinq caractéristiques principales sont : (i) l'expansion sociétale du recours au terrorisme, (ii) l'auto-radicalisation sur le mode héroïco-punitif, (iii) l'usage actif 
et innovateur des technologies de l'information, (iv) la symbiose entre la violence physique et la violence symbolique se déplaçant du champ de bataille à l'espace de bataille, et (v) l'auto-capacitation organisationnelle.

Aussi, dire la violence politique contemporaine, c'est désormais cerner l'enchâssement de questions évolutives dont les hybridités et les temporalités sont légion. Or, une pensée unique est bel et bien trop facilement venue enserrer la question du terrorisme. La discussion de ce qu'il recèle, d'où il provient et ce qu'il traduit a été simplifié. Les contradictions de ce storytelling trop huilé et en apparence désidéologisé abondent. Partant, la sous-conceptualisation de l'État islamique est la fille de la sousthéorisation d'Al Qaida, et ces deux déficits, se donnant la main d'une décennie l'autre, sont le pendant du marketing réussi médiatiquement et politiquement d'une guerre contre le terrorisme qui préside encore invisiblement aux destinées d'une néopensée unique. À ne parler que «terrorisme», "sécurité », «radicalisme», «extrémisme » et «jihadisme », l'université a perdu de vue l'essentiel, à savoir l'analyse de l'histoire, l'élaboration de la conceptualisation clinique si centrale aux sciences sociales et plus simplement le sens de ces changements.

Arc-boutés de la sorte sur les questions de définition (otage d'une subjectivité qui préside en la matière), de religion (seul le terrorisme islamiste/islamique est visible) et de «nouvelle vague» (chaque nouvel épisode serait indicateur d'un «profil»), nous avons volontairement appauvri notre compréhension d'un des phénomènes sociopolitiques les plus importants de notre époque. À penser le terrorisme au singulier, sous le prisme d'un reductio ad islamum et d'une incessante nouveauté, de telles approches ont privé états et sociétés d'outils plus intelligents pour faire sens des nuances nécessaires, mais elles ont surtout assis un anhistoricisme fort problématique. Au lieu de s'enfermer dans un récit réductionniste, désormais trop familier, sur « le terrorisme », un narratif appauvrissant notre capacité à conceptualiser la nature des formes de violences contemporaine, il faut plutôt construire analytiquement et mettre à jour des matrices de lectures face à des nouvelles formes de conflictualités postmodernes et post-mondialisées qui nécessite une historicisation plus élaborée que le narratif sécuritaire.

Al Qaida hier et l'EI aujourd'hui pointent du doigt une nécessaire relecture de la conceptualisation des relations internationales face à une pratique de la violence objectivement nouvelle - à savoir la délocalisation d'une projection de la violence transnationalisée. La matérialisation de l'État islamique est une indication d'une transformation en cours des relations internationales, non pas une aberration au milieu d'un ordre international qui serait simplement perturbé. La question n'est donc pas comportementale (l'EI en tant qu'identité) mais stratégique (l'EI en tant que mode opératoire). Au cœur de cette grammaire changeante des relations internationales émerge la question de la chose étatique et de son contre-champ non étatique. Ces entités et agents qui se multiplient de loin en loin occupant tour à tour territoires ou espaces virtuels, le tout de façon fluide. Et cette hybridité intrinsèque - territorialité et virtualité, poursuite du local et du transnational - est à la fois la force et la faiblesse de l'État islamique.

$\mathrm{Au}$ final, les circulations des contre-interrogations de cette violence émergente décuplent, enfin, l'acuité des conséquences de la réponse étatique. Le terrorisme ne se réduit pas à un face-à-face entre autorités et terroristes. Il s'est répandu dans les vies de tous et déborde sur d'autres thématiques, telle la question migratoire et celle des inégalités sociales et politiques. À la faveur d'actes de portée mondiale et de médiatisation extrême, les questions d'efficacité et de résultats ont, de façon 
stérilisante, pris le dessus fermant la porte au débat sur la sécurisation en cours qui génère une demande autoritariste rationalisée par la malléabilité de l'argument de « sales guerres ». Restituer le substrat postcolonial évanescent, insérer l'émergence du transnational post-moderne et interroger les effets sociétaux diffus du toutsécuritaire sont parmi les projets qui peuvent concourir à ensemencer un répertoire de significations plus riche des trajectoires de désintégration et de désintermédiation de la violence contemporaine au-delà des histoires d'Al Qaida et de l'État islamique. 